\title{
Microstructural Analysis of Radiation Defect in Ion-Irradiated Austenitic Stainless Steel using TEM and 3D-APT
}

\author{
Hyung-Ha Jin ${ }^{1}$, Eunsol Ko ${ }^{1}$, Sangyeop Lim ${ }^{1}$, Junhyun Kwon ${ }^{1}$, Bongho Lee ${ }^{2}$ \\ ${ }^{1}$ Nuclear Materials Research Division, Korea Atomic Energy Research Institute, 989-111 \\ Daedeok-daero, Yuseong-gu, Daejeon, 305-353, Republic of Korea \\ ${ }^{2}$ National Center for Nanomaterials Technology (NCNT), Pohang University of Science and \\ Technology (POSTECH), 77 Cheongam-ro, Nam-gu, Pohang, Gyeongbuk, 790-784, Republic of Korea
}

Austenitic stainless steel (ASS) used for internal components in a nuclear reactor is exposed to severe neutron irradiation at high doses (up to $100 \mathrm{dpa}$ in a pressurized water reactor). Microstructural changes by neutron irradiation are known to lead to material degradation such as radiation hardening and irradiation assisted stress corrosion cracking (IASCC) during operation [1-3]. The formation of radiation defects and the development of solute segregation at the grain boundary have been considered as typical radiation induced phenomena to affect the material degradation in an ASS. Since radiation hardening is strongly correlated with the evolution of radiation defects, extensive studies have still been progressed to understand the characteristics of a radiation defect in irradiated ASS using various analytical techniques such as transmission electron microscopy (TEM) and 3D-atom probe tomography (3D-APT). In this work, we focused on the change in characteristics of radiation defects with irradiation temperature. We used ion irradiation to produce the simulated material, and performed a TEM examination and 3D-APT analysis to reveal the characteristics of the radiation defect in the ion-irradiated ASS.

First, we prepared experimental samples by ion irradiation at different irradiation temperatures. The ion irradiation experiment was carried out with a Tandem ion accelerator in the Korea Institute of Geoscience \& Mineral Resources (KIGAM). We used iron ions $\left(\mathrm{Fe}^{4}+\right)$ with an energy of $8 \mathrm{MeV}$. The ion dose is approximately $5.6 \mathrm{E} 15 \mathrm{ion} / \mathrm{cm}^{2}$. The irradiation experiments were performed at $473 \mathrm{~K}, 573 \mathrm{~K}$, and $673 \mathrm{~K}$, respectively. Radiation damage is calculated to be formed up to about 2 um in depth by the Stopping Range of Ions and Matter (SRIM) code. TEM analysis for the radiation defects was conducted at a depth of around $1 \mathrm{um}$. A high density fine frank loop was observed in the ion irradiated austenitic stainless steel. The results of the TEM analysis of the irradiated samples are shown in Figure 1. As the irradiation temperature increased, the average size of the frank loop increased while its number density decreased. In the experimental sample irradiated at $674 \mathrm{~K}$, unidentified phases with a few nanometers in size were also observed in the matrix, which were taken at near the $<100>$ zone axis. The depletion of $\mathrm{Cr}$ and the enrichment of $\mathrm{Ni}, \mathrm{Si}$, and $\mathrm{P}$ (Radiation induced segregation, RIS) were observed at high angle grain boundaries in the ion-irradiated sample. The RIS phenomenon was strongly detected after ion irradiation at $673 \mathrm{~K}$. We conducted a 3D-APT analysis of the ion-irradiated samples irradiated at $573 \mathrm{~K}$ and $673 \mathrm{~K}$ to show chemical instability with irradiation temperature. The 3D-APT analysis in Figure 2 indicates that the solute distribution is heterogeneous in the measured volume. The enrichment of $\mathrm{Ni}$ and $\mathrm{Si}$ in the atomic map is considered to be the segregation at the frank loop. The segregation of $\mathrm{Ni}$ and $\mathrm{Si}$ was clearly detected near the faulted loop after the ion irradiation at $673 \mathrm{~K}$ while it weakened after 573 $\mathrm{K}$ irradiation. The RIS at the frank loop is dependent on the irradiation temperature. The frank loop is thought to be one of the potential sites for strong RIS phenomenon as well as high angle grain boundary. 


\section{References}

[1] E. P. Simonen, S.M. Bruemmer, JOM. 50 (1998) 52-55.

[2] E. A. Kenik, J.T. Busby,Mater. Sci. Eng. R. 73 (2012) 67-83.

[3] S.M. Bruemmer et al, J. Nucl. Mater. 274 (1999) 299-314.

[4] This research was supported by the National Research Foundation of Korea (NRF) grant funded by the Korea government (MSIP) (No. 2012M2A8A4025886).
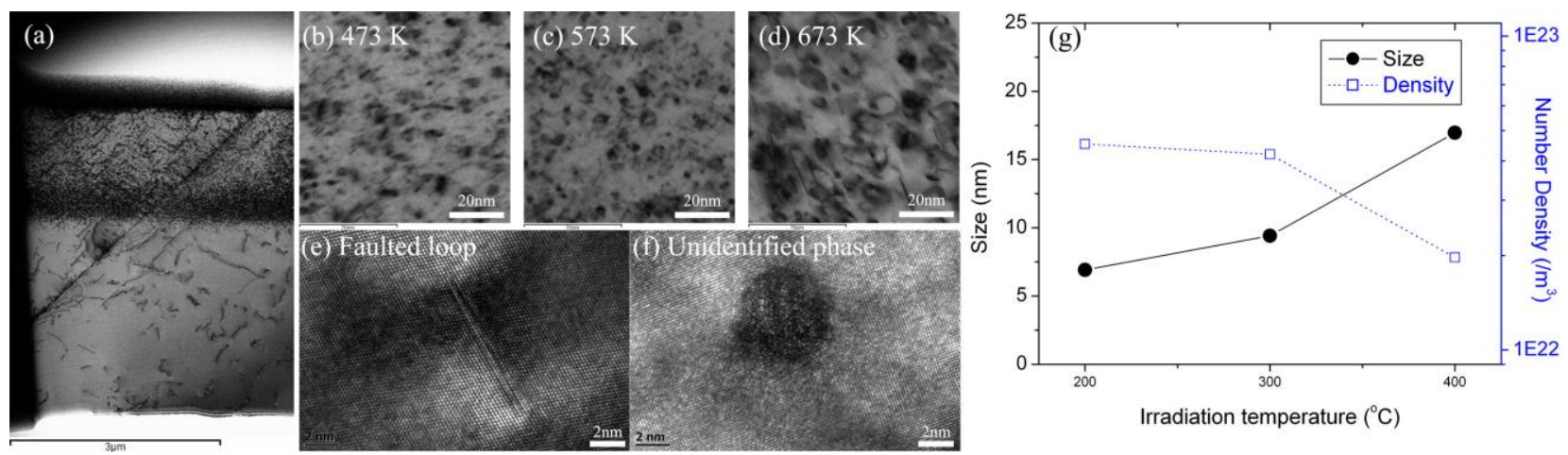

Figure 1. A Low magnified TEM image showing damage layer introduced by the ion irradiation (a), TEM images showing radiation defects after ion irradiation at $473 \mathrm{~K}$ (b), $573 \mathrm{~K}$ (c) and $673 \mathrm{~K}$ (d), a HRTEM image of typical frank loop (e) (faulted loop with a Burgers vector $\mathrm{a}_{0} / 3<111>$ lying in $\{111\}$ planes) after the $673 \mathrm{~K}$ irradiation and a HRTEM image of an unidentified phase (f) taken at zone axis of $<100>$ in experimental sample after ion irradiation at $673 \mathrm{~K}$. The average size and the number density of frank loop measured by TEM analysis is given in $(\mathrm{g})$.
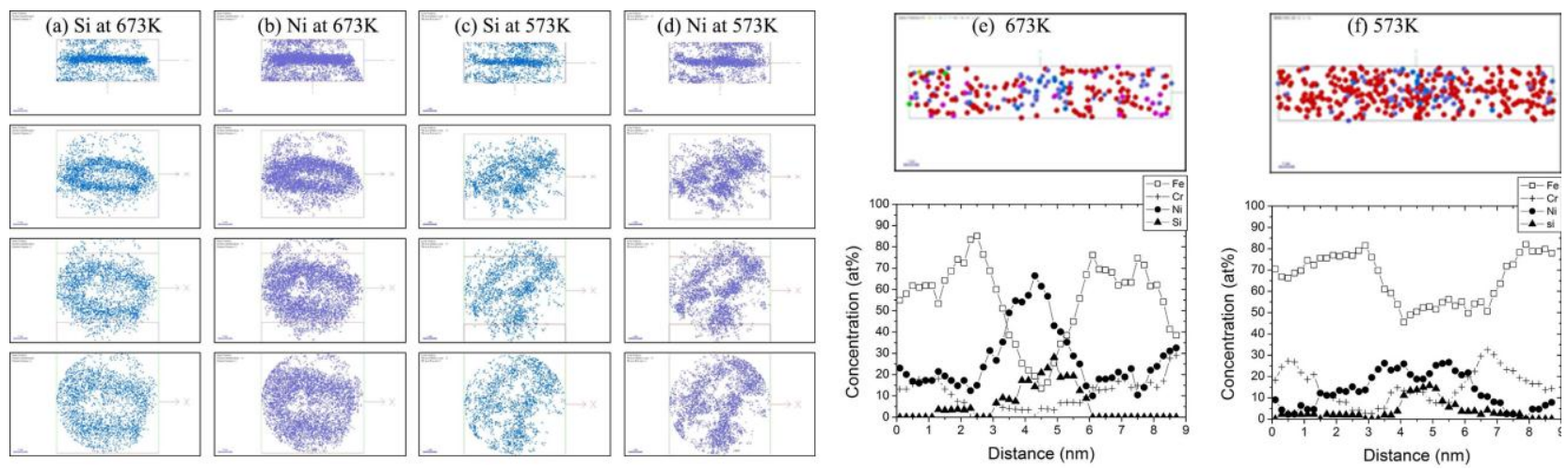

Figure 2. Atomic maps of $\mathrm{Si}$ and $\mathrm{Ni}$ atoms showing the segregation at dislocation loop in the austenitic stainless steels irradiated at $673 \mathrm{~K}(\mathrm{a}, \mathrm{b})$ and $573 \mathrm{~K}(\mathrm{c}, \mathrm{d})$. Concentration profiles of each element through the dislocation loop observed after the ion irradiation at $673 \mathrm{~K}$ (e) and $573 \mathrm{~K}$ (f). 